\title{
El principio de integración medioambiental dentro de la Unión Europea: la imbricación entre integración y desarrollo sostenible
}

\author{
Susana Aguilar Fernández
}

Universidad Complutense de Madrid. Facultad de Ciencias Políticas y Sociología

Susana@ceacs.march.es

\section{Resumen}

El principio de integración, tal y como fue inicialmente concebido por la UE, se define como la incorporación del componente medioambiental a todas aquellas políticas con efectos negativos sobre el entorno, siendo su principal objetivo mejorar los rendimientos de la política de protección del medio ambiente a nivel comunitario. Posteriormente, este mismo principio se ha vinculado a la estrategia europea de desarrollo sostenible (DS), que propugna la compatibilidad entre crecimiento económico, conservación del medio físico y bienestar social. La vinculación del principio de integración al DS se ha traducido en lo que podría denominarse una "versión ampliada» (u horizontal) del mismo, que aspira a impregnar el proceso político vinculado al programa de sostenibilidad ${ }^{1}$ en su totalidad, desde la etapa inicial de formación de agenda hasta la etapa final de evaluación de resultados, frente a la inicial «versión restringida» (o vertical), que se centraba meramente en la aplicación de criterios medioambientales al funcionamiento de determinados organismos públicos. Como la teórica armonía que se proclama con respecto a los tres elementos que componen el DS es falsa, el principal problema de esta versión ampliada es que oculta los importantísimos conflictos de interés que se producen cuando se intenta llevar el DS a la práctica. La difícil aplicabilidad de la sostenibilidad se refleja, entre otras cosas, tanto en que la dimensión horizontal del principio de integración, plasmada en las estrategias de DS que han elaborado o todavía están elaborando muchos países de la OCDE, no ha sido (o sólo insuficientemente) desarrollada, como en que la dimensión vertical se ha consolidado más en el nivel retórico-formal de la política que en el sustantivo.

Palabras clave: principio de integración, desarrollo sostenible, Unión Europea, política medioambiental.

\section{Abstract. The principle of environmental integration in the European Union: the interlocking between integration and sustainable development}

The integration principle was initially conceived by the EU as the incorporation of environmental concerns into those policies with harmful effects on the environment, its main aim being the improvement of Community environmental policy. Later on, the same principle has been linked to the European Strategy of Sustainable Development (SD) which advocates the compatibility between economic growth, environmental conservation and

1. En este trabajo se entiende, meramente por razones de estilo (esto es, para evitar repeticiones), que DS y sostenibilidad son sinónimos. 
social welfare. This connection has reflected itself in a "global version» (horizontal) of the principle, in the sense that the sustainability programme aspires now to influence the entire political process, from its early stage of agenda-setting to the final stage of policy assessment, in contrast with the «narrow version» (vertical) which simply revolved around the need to apply environmental criteria to the working of certain public agencies. The main problem of the global version is that it blurrs the crucial conflicts of interest that are brought about when SD is put into practice. This is so because the theoretical harmony between the three different pillars which compose SD is false. The low political feasibility of SD can be seen in the insufficient development of the horizontal dimension of the integration principle, as shown by the national strategies of SD which are underway in the OECD, and also in the fact that the vertical dimension of the principle is adhered to in a rather rethorical way.

Key words: integration principle, sustainable development, European Union, environmental policy.

\section{Sumario}

1. El principio de integración en los textos internacionales: la imbricación de la integración y el desarrollo sostenible

2. El principio de integración y la necesidad de new governance asociada al reto de la sostenibilidad
4. El principio de integración en el entramado europeo

5. Conclusiones

Bibliografía

3. Formas de entender el principio de integración

\section{El principio de integración en los textos internacionales: la imbricación de la integración y el desarrollo sostenible}

Aunque el principio de integración, entendido como la necesidad de incorporar el componente medioambiental en todas aquellas políticas que tienen efectos negativos sobre el entorno con el objetivo de mejorar los resultados de la política de medio ambiente (o de reducir su déficit de implementación), ha tenido una larga e importante presencia dentro de la Unión Europea, se ha visto reforzado recientemente a raíz de la aprobación de la denominada «Iniciativa de Cardiff» y de su vinculación a la estrategia comunitaria de desarrollo sostenible (DS). Ya el Tercer Plan de Acción Medioambiental (PAM), que cubría el periodo 1982-1986, defendió la necesidad de integrar el medio ambiente en las políticas sectoriales, mientras que en la última reforma de los textos fundacionales europeos, plasmada en el Tratado de Amsterdam, de 1997, se señala, en el artículo $6^{\circ}$, que «los requerimientos de la protección medioambiental deben ser integrados en la definición e implementación de las distintas políticas de la Comisión [...] principalmente con el objetivo de promover el DS». Por otro lado, el Informe Brundtland (Our Common Future), de 1987, que consagra el concepto de DS a nivel internacional, afirma, en su capítulo $12^{\circ}$, que una de 
las aproximaciones con respecto a la cuestión medioambiental debe centrarse en las políticas que son la fuente de efectos sobre el medio ambiente, así como que la capacidad para elegir políticas que son sostenibles exige que las dimensiones ecológicas sean consideradas al mismo tiempo que las económicas, comerciales, energéticas, agrícolas, industriales y otras. Este es, según el informe, el principal reto institucional de la década de los 90: adaptar las prácticas de gobierno al DS, haciendo del principio de integración uno de los mecanismos clave de tal adaptación. Asimismo, y dentro de la Agenda 21 (capítulo $8^{\circ}$ ), que dimana de la Cumbre sobre Desarrollo y Medio Ambiente auspiciada por la ONU en Río de Janeiro en 1992, se señala la necesidad de integrar el medio ambiente y el desarrollo en el proceso decisorio y en los niveles de planificación y gestión, y también de establecer sistemas integrados de contabilidad medioambiental y económica. En el ámbito de la OCDE, por último, el principio de integración está también estrechamente vinculado al DS, ya que se le considera un instrumento básico a la hora de afrontar el principal reto de la sostenibilidad, que no es otro que el de disociar (de-coupling) el crecimiento económico de las presiones negativas sobre el medio ambiente que históricamente le han acompañado (Lafferty, 2002). En nuestros días, los conceptos de integración y DS están, por lo tanto, inextricablemente imbricados.

Un breve repaso a los hitos más recientes que han acompañado al principio de integración en la UE podría comenzar en 1997, cuando el Consejo Europeo decide, a propuesta del primer ministro sueco, Gorän Persson, pedir a la Comisión que elabore propuestas para implantar el mecanismo de integración y así avanzar en el camino hacia el DS. Siguiendo en esta misma línea se sitúa el «Proceso de Cardiff», que fue iniciado por la Cumbre de Luxemburgo, en diciembre de 1997, aunque adquirió rango de programa comunitario en la siguiente reunión, en Cardiff, en junio de 1998. La también denominada «Iniciativa de Cardiff» se ha ido perfilando en las cumbres de Viena, Colonia y Helsinki, y se ha plasmado gradualmente en una rutina según la cual todas las instancias relevantes del Consejo se comprometen a desarrollar una estrategia de progreso en materia de integración y DS en sus respectivos ámbitos, así como a rendir cuentas en términos de los avances o retrocesos producidos en la misma. Aunque inicialmente este compromiso iba dirigido a tres sectores clave (transporte, energía y agricultura) y a sus respectivas direcciones generales en la Comisión, el mandato se ha ampliado posteriormente a otras políticas: mercado interno, ayuda al desarrollo, industria y empresa, ssuntos generales, economía y finanzas, y pesca. En un reciente informe de evaluación se señala que puede caracterizarse el Proceso de Cardiff como vinculante (binding) y de compromiso (committing): aunque «legalmente, su naturaleza vinculante es bastante débil, el compromiso político es fuerte. Existió una clara voluntad política al comienzo que fue reforzada, en distintos niveles, a lo largo de todo el proceso. De especial importancia resultan los distintos compromisos alcanzados por el Consejo para mejorar y revisar las estrategias, y los encargos globales (work packages) delegados a la Comisión o a específicos grupos de trabajo» (Krämer, en Lafferty, 2002, p. 6). 
Los jefes de estado y gobierno de la UE mantuvieron el compromiso alcanzado en Cardiff en la Cumbre de Helsinki, de 1999, en la que el Consejo pidió a la Comisión que propusiera una estrategia europea de DS. La Comisión, en el año 2001, elaboró una comunicación en la que delineaba retos, principios clave, prioridades y objetivos concretos del DS, y en ese mismo año acordó su 60 PAM, titulado "Medio Ambiente 2010: Nuestro Futuro, Nuestra Elección» (Environment 2010: Our Future, Our Choice) $)^{2}$. El $6^{\circ}$ PAM se centra principalmente en temas procedimentales, o en lo que la actual comisaria de Medio Ambiente, Margo Wallström, ha denominado las tres «ies»: integración, implementación e información. Asimismo, este PAM incorpora la necesidad de revisar regularmente el progreso que se produce en la integración sectorial del medio ambiente. Por otro lado, la Estrategia Europea de Desarrollo Sostenible, que fue presentada al Consejo en la Cumbre de Gotenburgo, en junio del 2001, remarca que «el proceso de integración de las preocupaciones (concerns) medioambientales en las políticas sectoriales, propulsado por el Consejo Europeo en Cardiff, debe continuar y proporcionar un input medioambiental a la estrategia comunitaria de DS similar a aquél dado a las dimensiones económicas y sociales que recogen las Guías de Política Económica Global (Broad Economic Policy Guidelines) y las Guías de Creación de Empleo (Employment Guidelines). Las estrategias de integración sectorial del medio ambiente deben ser coherentes con los objetivos específicos de la estrategia de DS de la UE» (en Lafferty, 2002 , p. 6). Así pues, los objetivos medioambientales y de DS ocuparon en Gotenburgo, por primera vez, un lugar significativo en la agenda del Consejo, al tiempo que se recalcaba, dentro del apartado de conclusiones, que un principio clave de la sostenibilidad es que «los efectos económicos, sociales y medioambientales de todas las políticas [sean] examinados de forma coordinada y tenidos en consideración en el proceso de toma de decisiones». En esta cumbre, asimismo, y tras aceptarse la Estrategia Europea de DS, los máximos dignatarios comunitarios se comprometieron a desarrollar una específica estrategia de sostenibilidad que contuviera objetivos específicos y mecanismos de evaluación de los mismos.

Uno de los problemas del principio de integración es que los documentos europeos, tal y como acaba de verse, consideran la integración de los objetivos medioambientales en cuestiones económicas y sociales en términos de equilibrio, compatibilidad y conciliación, pero no hablan en ningún momento de dar prioridad al componente medioambiental, lo cual, según autores como

2. A pesar de la relevancia mediática del DS y de las declaraciones a favor de la sostenibilidad que se han producido tras diversas cumbres del Consejo Europeo, es ésta una tarea en la que no se ha avanzado grandemente, tal y como mostró el informe de la Agencia Europea de Medio Ambiente (AEM), «El Medio Ambiente en la Unión Europea en el Umbral del Siglo XXI», publicado y presentado a los ministros de Medio Ambiente en junio de 1999. Este juicio se ha confirmado en el informe de mayo del 2000, «Señales Medioambientales 2000», que, elaborado también por la AEM, incluye más de ochenta indicadores medioambientales, que acompañarán al PIB y se aplicarán anualmente. 
Lafferty (2002), es imprescindible si de lo que se trata es de avanzar hacia el DS en los países desarrollados. Aunque esta priorización es cuestionable, sí es cierto que el principal reto para la institucionalización del principio de integración es su incorporación real a las políticas económicas, ya que la UE ha apostado por una estrategia de DS en la que la protección medioambiental y el freno al deterioro del entorno dependen, en gran medida, de la disponibilidad de medios materiales y de la consecución del crecimiento económico. En la comunicación de la comisión titulada "Hacia una Cooperación Global» (Towards a Global Partnership), se pone el énfasis en que «las fuerzas del mercado deben ser aprovechadas para mantener y aumentar el crecimiento y para crear puestos de trabajo, al tiempo que se preserva el medio ambiente para las generaciones futuras y se fortalece la cohesión social»; asimismo, se recalca la necesidad de «asegurar que la globalización contribuya al DS eliminando las distorsiones comerciales» (Bomberg, 2002).

\section{El principio de integración y la necesidad de new governance asociada al reto de la sostenibilidad}

Aunque algunos autores han considerado que la proteccion medioambiental es una de las políticas de mayor éxito dentro de la UE (Collier, en Grant y otros, 2000), este éxito debe ser entendido más desde un punto de vista político global (politics), ya que el apoyo de los ciudadanos a la actuación comunitaria en esta materia es muy alto y por ello la política de medio ambiente ha funcionado indirectamente como motor del proyecto europeo, que desde el punto de vista de la política específica (policy). Es decir, a pesar de que la política comunitaria medioambiental ha introducido rigor y novedosos planteamientos incluso en las agendas de aquellos países más comprometidos con la resolución del problema ecológico, su déficit de implementación, entendido como la distancia entre objetivos y resultados, es muy grande. Según declaraciones de la propia Comisaria de Medio Ambiente, si no se hace nada por resolver el déficit de implementación, éste «se convertirá rápidamente en un problema de credibilidad" (credibility gap).

Sin entrar ahora a discutir las razones de este déficit, su generalizada constatación ha provocado un debate acerca de la necesidad de una nueva governance en política medioambiental ${ }^{3}$. Por governance se entiende «un proceso

3. El déficit medioambiental está vinculado a diferentes factores, algunos de los cuales son específicos del país en cuestión (obedecen, por ejemplo, a tradiciones legales concretas), mientras que otros son universales (la "traslación de problemas", o problem-dislocation, que se refleja en que las mejoras en las políticas atmosféricas y de aguas continentales hayan agravado los problemas de gestión de residuos en todos los países, es el mejor ejemplo). De los factores explicativos del déficit de implementación medioambiental destacan los siguientes: la indefinición de las políticas, o políticas que no identifican adecuadamente los propósitos y las acciones, o que proporcionan insuficientes incentivos a los actores a los que van dirigidas; los conflictos de jurisdicción, o problemas que surgen cuando distintas agencias dentro del mismo nivel comparten competencias con respecto a la implementación; las trampas 
público no centralizado que [intenta llegar a un] equilibio entre libertad y orden con vistas a una gestión democrática, y que incluye, pero no está limitado a, las actuaciones de los gobiernos y sus burocracias» (Rayner, 1994, p. 5). Elementos integrantes de esta nueva governance serían, junto al principio de integración, los de precaución, coordinación, subsidiariedad, participación y transparencia, y rendimiento de cuentas (accountability). «Debido a la naturaleza muchas veces cambiante, interdisciplinaria, transectorial y transfronteriza de muchos problemas medioambientales actuales (la lluvia ácida es uno de los mejores ejemplos), está surgiendo una nueva governance basada en pautas de cooperación funcional (entre las autoridades y los grupos sociales) y territorial (entre las autoridades nacionales, regionales y locales), que mezcla distintos tipos de instrumentos políticos y va más allá de los tradicionales enfoques regulativos impuestos desde arriba (top-down) y centrados en "ordenar y controlar" (command-and-control), y que muestra un mayor interés por el cumplimiento y la evaluación de las políticas (policy enforcement y policy assessment) que por la producción y reforma legislativa» (Aguilar, 2002, p. 8).

Uno de los principales retos que debe afrontar una nueva governance basa$\mathrm{da}$, entre otros, en el principio de integración, es que tiene que competir con formas de gobernar caracterizadas por inercias y malos hábitos fuertemente enraizados en tradiciones políticas y culturas organizativas, tales como la «lealtad institucional que alimenta la fragmentación y la rivalidad entre y dentro de las instituciones» (Bomberg, 2002, p. 8). Es bien sabido que los burócratas generalmente apoyan aquellas estrategias que maximizan los beneficios de aquellas instituciones dentro de las que trabajan: estrategias que persiguen no sólo la asignación de mayor presupuesto (dentro de la dinámica incrementalista que impera en la administración), sino, principalmente, la acumulación de poder. Este poder puede ser entendido como el manejo de más cantidad de recursos no únicamente económicos, sino, sobre todo, políticos: de estatus o prestigio, y de discreción o margen de maniobra. A la hora de luchar por la distribución de estos recursos, las instituciones se ven a sí mismas inmersas en un duro mercado de competencia con otras instituciones a las que consideran rivales, lo cual no favorece, indudablemente, la puesta en práctica de principios que, como el de integración, promueven la coordinación y cooperación en el desarrollo de políticas que tradicionalmente

de la decisión conjunta (joint-decision traps), cuando determinadas competencias están bajo la responsabilidad de distintos niveles político-administrativos y ello conduce a procesos decisorios interminables e ineficientes; la insuficiente integración del componente medioambiental en otras políticas; el énfasis en el perfeccionamiento de la legislación, que provoca una "saturación" de textos, al tiempo que se relega a un segundo plano la necesidad de hacer cumplir lo ya aprobado (policy enforcement); las diferencias en la interpretación de la política, cuando los funcionarios regionales o locales encargados de aplicar la legislación aprobada en el ámbito nacional reducen la exigencia de la ley en su relación cotidiana con los agentes encargados de cumplirla, y la captura de agencia (agency-capture), que ocurre cuando determinados organismos públicos se convierten en «rehenes» de los poderosos grupos de interés a los cuales teóricamente deben regular (Aguilar, 2002). 
han sido elaboradas desde el prisma sectorial y fragmentado del ministerio al que pertenecen.

\section{Formas de entender el principio de integración}

Existen dos maneras genéricas de entender el principio de integración: la primera es anterior a la primacía que, principalmente desde finales de los años 80 , ha conseguido el concepto de DS y se centra en integrar el componente medioambiental en todas aquellas políticas sectoriales con efectos negativos sobre el entorno como forma de reducir el déficit que acompaña a la política de medio ambiente (esto es lo que podría denominarse la «integración restringida»); la segunda es posterior en el tiempo y está inextricablemente vinculada al DS, ya que postula que en la sostenibilidad hay una interdependencia, y debe haber un equilibrio, entre los componentes medioambientales, económicos y sociales (a esto se le podría etiquetar de «integración ampliada»). En esta segunda acepción el énfasis se pone en la necesidad de que el crecimiento económico integre (o se vea acompañado por) tanto la preocupación medioambiental, de forma que se reduzcan sus externalidades sobre el medio físico, como la preocupación social, de forma que el bienestar material se distribuya de forma menos desigual. Sólo así el crecimiento económico se sostendrá en el tiempo, porque, al generar cohesión social y mejorar la conservación del entorno, gozará de legitimidad entre la ciudadanía. Mientras que en el caso de la integración restringida no era necesario hablar de prioridades, ya que lo único (aunque evidentemente complicado) que había que conseguir era que los distintos responsables de las políticas incorporaran el componente medioambiental en la elaboración y el desarrollo de sus propuestas, en la integración ampliada se puede discutir sobre qué elemento debe ser prioritario: cuánto crecimiento y a qué precio; qué desarrollar más, las políticas de bienestar o las de medio ambiente, por ejemplo. Es en este contexto en el que algunos autores han defendido que los tres elementos del DS no tienen por qué ser tratados de la misma manera y que, de hecho, tal y como se señaló anteriormente, el elemento medioambiental es el fundamental, ya que sin éste «el concepto pierde su especificidad» (Lafferty, 2002, p. 2). Esta aseveración puede ser justificada, principalmente, en el contexto de aquellos países desarrollados en los que los estados de bienestar y las políticas sociales han tenido (con distintos grados de eficacia, por supuesto) efectos amortiguadores en cuanto a la distribución desigual de la riqueza, contribuyendo así a generar un cierto consenso en torno a las bondades del sistema de mercado. No es sin embargo fácilmente justificable en el caso de los países en vías de desarrollo, en los cuales, debido, entre otras muchas razones, a sus inexistentes o deficientes estados de bienestar, el crecimiento económico (si es que se ha producido) no ha contribuido a mejorar el nivel de vida de la mayoría de los ciudadanos. En este último caso parece lógico pensar que el componente social del DS esté por encima del medioambiental, aunque esta elección responda indudablemente a un planteamiento simplista, de corto plazo y electoralista de la política, y no al planteamiento 
complejo (por la interdependencia que subyace en el concepto de sostenibilidad), de largo plazo (o intergeneracional) y de consenso partidista e institucional que caracteriza, o debería caracterizar, al DS.

Mas allá de esta distinción genérica entre integración restringida y amplia$\mathrm{da}, \mathrm{y}$ a pesar de la presencia del principio en todo tipo de documentos desde hace ya más de dos décadas, puede afirmarse que el concepto de «integración política medioambiental» (environmental policy integration) no ha sido desarrollado adecuadamente y que no existe unanimidad con respecto a su significado. Como prueba de ello, un reciente manual de política medioambiental recalcaba lo siguiente: «a pesar del compromiso progresivo en favor de la integración del medio ambiente, se ha prestado relativamente poca atención a la definición del concepto. Existe una confusa variedad de métodos a la hora de tener más en cuenta los factores medioambientales en el desarrollo de las políticas sectoriales» (Lafferty, 2002, p. 6). Puesto que aquí se ha defendido la imbricación actual entre integración y sostenibilidad, la discusión que sigue a continuación sobre formas de entender el principio hay que situarla dentro de la versión ampliada de la integración ${ }^{4}$.

Algunos autores, como Liberatore, han distinguido, de manera global, la integración con respecto a temas (issues), sectores, organización, espacio (cooperación internacional) y tiempo (solidaridad intergeneracional), elementos distributivos e instrumentos políticos. Collier, por otro lado, señala que los objetivos de la integración de la política medioambiental son tres: la consecución del DS y la prevención del daño medioambiental, la eliminación de contradicciones entre las políticas así como dentro de las mismas, y el entender los beneficios mutuos que se derivan de intentar que las políticas se apoyen entre sí. De estos tres objetivos, tal vez sea el segundo el que apunta con mayor precisión al principal significado del principio de integración, ya que éste no consiste sino en la necesidad de introducir coordinación y coherencia en la elaboración de las políticas. El tercero aporta un elemento normativo (entendido como que de la integración se derivan beneficios para todas las políticas) que no tiene por qué ser necesariamente cierto, a menos que se adopte un punto de vista simplista, o idealista, que defienda la total armonía entre los tres elementos que configuran el $\mathrm{DS}^{5}$, o una perspectiva de ecologismo radical, para la cual la protección del medio ambiente sea un bien por encima de cualquier otro. Ello es así porque este elemento normativo oscurece el ineludible juego político, con la aparición de ganadores y vencedores, que acompaña a las estra-

4. La discusión que sigue se basa principalmente en Lafferty (2002).

5. Junto al enfoque armónico está aquél otro que postula el equilibrio entre los tres elementos del DS. En el estudio de Collier sobre la integración del componente medioambiental en la política energética europea, las preocupaciones medioambientales, económicas y energéticas se presentan como equilibradas, ocupando cada una de ellas los vértices de un triángulo dentro del cual está el principio de integración. En muchos casos, este equilibrio es indefendible, porque ciertos objetivos políticos o económicos implican un reto enorme para la pervivencia de determinados ecosistemas y pueden provocar su pérdida irreparable (Lafferty, 2002). 
tegias de sostenibilidad ${ }^{6}$. Por otro lado, que estos beneficios universales no son ni mucho menos evidentes para los que toman las decisiones en el gobierno y la administración puede ser una de las razones por las cuales existen palpables resistencias a la hora de operacionalizar el principio de integración.

Según Underdal, para que una política (no necesariamente la medioambiental) esté integrada tiene que cumplir tres requisitos: comprehensividad (que incorpore ámbitos temporales y espaciales, actores y temas), agregación (que sea evaluada desde una perspectiva global) y consistencia (que los diferentes elementos de la política estén en consonancia recíproca). La definición de política integrada sería aquella en la que: «todas las consecuencias relevantes de las decisiones políticas son reconocidas como premisas de la decisión, en la que todas las opciones políticas son evaluadas sobre la base de sus efectos según algún indicador agregado de utilidad, y en la que los diferentes elementos de la política son coherentes entre sí» (Lafferty, 2002, p. 9). Si se traslada esta definición al principio de integración medioambiental y se combina con el análisis de políticas públicas, se obtiene lo siguiente:

- La integración del componente medioambiental debe producirse en todas las fases del proceso decisorio de las políticas sectoriales: desde la fase de formación de la agenda (agenda-setting) hasta la encargada de la evaluación. El paralelismo de esta integración total se encontraría en la política económica, cuyos principios básicos (tales como el equilibrio presupuestario, el control de la inflación, las bajas tasas de interés, etc.) informan actualmente todas las decisiones tomadas en las distintas áreas de gestión pública, debido, entre otras cosas, a la férrea tutela que ejercen los ministerios de Hacienda y de Economía y al consenso internacional acerca de la necesidad de aplicar una determinada ortodoxia económica. Hipotéticamente, algo parecido podría ocurrir, por ejemplo, con respecto a un principio medioambiental tan básico como es el del ahorro de agua, si se aplicara una estrategia que estableciera objetivos concretos a cumplir en plazos determinados por distintas instancias (obviamente, el Ministerio de Agricultura sería el primer destinatario, pero también el de Fomento, por poner otro caso, que debería mejorar ciertas infraestructuras hidráulicas que dilapidan el recurso, o justificar nuevas obras que quedarían en entredicho dentro de una campaña que pone límites a la demanda de agua) que se comprometerían, a su vez, a coordinar sus políticas entre sí, a evaluar sus resultados y a hacerlos públicos.

6. El DS implica tomar decisiones acerca de prioridades políticas y tiene, ineludiblemente, como resultado la aparición de ganadores y perdedores. Por ello, algunos autores han alertado sobre la simplificación que supone el considerar al DS como un juego de suma positiva, o winwin game (Lafferty, 2002), que ignora su naturaleza política y distributiva. La caracterización de la sostenibilidad como juego de suma positiva sólo es cierta, teóricamente, si se tiene en cuenta a las generaciones futuras al tiempo que se relega a un segundo término a los grupos que, en el presente, tendrán que soportar el peso de las decisiones que conducen al DS. 
- En los casos en los que exista conflicto de intereses entre la integración del componente medioambiental y las prioridades u objetivos tradicionales de las distintas políticas, debería darse prioridad al principio de integración, y, si esto no fuera posible, deberían hacerse explícitos los criterios que han conducido a la decisión contraria al mismo. Aquí se vuelve de nuevo a la idea conexa según la cual el objetivo de protección del medio ambiente debería, para algunos autores, ser prioritario en relación con los otros dos, de tipo más "clásico", que componen el DS: el bienestar social y el crecimiento económico, ya que, para los gobiernos de los países desarrollados, el considerar los temas económicos y sociales como clave dentro de sus agendas, relegando el problema de las consiguientes externalidades sobre el entorno a la categoría de asunto secundario a tratar en un momento posterior, resulta relativamente sencillo e implica seguir con la forma de hacer política que tradicionalmente se ha venido haciendo (business as usual), caracterizada por sus grandes fracasos a la hora de mejorar la conservación del medio físico (Aguilar, 2002).

La integración del componente medioambiental en todas las fases del proceso decisorio, o integración total, se corresponde con la dimensión horizontal del principio. La dimensión horizontal denota el alcance en que una determinada autoridad a nivel central, que puede ser el propio gobierno, o bien una comisión o agencia específica a la cual se ha asignado una responsabilidad global de aplicación del DS, o un ente interministerial (al estilo del Grupo-Prodi para la estrategia de DS, dentro de la Comisión Europea), ha desarrollado una estrategia intersectorial comprehensiva. Esta dimensión del principio de integración define un proceso caracterizado por un fuerte elemento de negociación entre distintas agencias públicas a través del cual se intenta que al componente medioambiental se le otorgue, al menos, la misma consideración que a otros intereses sectoriales. En esta negociación entre distintos ministerios y sectores sociales que persiguen distintos objetivos, o incluso objetivos medioambientales en competencia, hay que hacer explícitas las consecuencias alternativas posibles que se derivan de distintas elecciones políticas, con sus potenciales efectos perversos (lo que los economistas denominan rebound effects, o los politólogos han etiquetado como problem-dislocation o Problemverschiebung). Una manera preliminar de evaluar el grado de desarrollo de la dimensión horizontal del principio es determinar qué organismo ha sido designado para aplicar el DS, así como cuál es la prioridad que se concede a la sostenibilidad y cómo se transmite la misma. Algunos de los mecanismos que ayudan a hacer operativa esta dimensión son:

- La existencia o creación de cláusulas constitucionales que definen el estatus especial de los objetivos medioambientales y de DS.

- Una estrategia de DS de largo plazo que, incorporando plazos y objetivos, se apoye sobre un claro mandato político y sea auspiciada por una figura clave dentro del ejecutivo. 
- La designación de un organismo específico de gobierno encargado de la coordinación global, aplicación y supervisión del proceso de integración.

- Una clara política de comunicación con respecto a las responsabilidades sectoriales para la consecución de los objetivos a través de los mecanismos de integración vertical (de los que se hablará más adelante).

- La evaluación periódica del progreso en cuanto a objetivos tanto a nivel central como sectorial.

- La existencia de mecanismos o cuerpos específicos para la resolución de conflictos de interés entre el objetivo medioambiental y los otros objetivos.

Frente a esta dimensión, existe también una integración vertical, entendi$\mathrm{da}$ en un sentido funcional e intradepartamental, que se refiere al alcance en que los objetivos medioambientales se han convertido en centrales para, y han sido aplicados por, un sector del gobierno, sea éste de ámbito nacional, regional o local. En una palabra, aquí se analiza hasta qué punto un determinado ministerio, departamento o consejería se ha «vuelto verde»: cómo ha mezclado el componente medioambiental con sus objetivos sectoriales tradicionales, de forma que en su funcionamiento cotidiano se opere con premisas de respeto ecológico ${ }^{7}$. Según este planteamiento, el primer componente no tiene por qué ser el dominante, ni tampoco, por supuesto, ser el principal a nivel del gobierno en su conjunto. La importancia que se conceda a la protección del medio ambiente depende tanto del compromiso político del ministro, o titular del organismo en cuestión, como de la habilidad de los burócratas a la hora de equilibrar ciertas presiones que, desde dentro, empujan en la dirección de asumir el nuevo principio, con las presiones que, desde fuera, empujan en la dirección contraria, dando prioridad a los objetivos que tradicionalmente se han defendido y que han beneficiado a las "clientelas de siempre». Los mecanismos para hacer operativa esta dimensión vertical son los siguientes:

— La identificación de los impactos ecológicos más importantes de la actividad sectorial, vinculados a actores y procesos e incluyendo al propio ministerio como origen de estos impactos (informe sobre el estado de la cuestión).

7. En la propuesta relativa a quince «objetivos nacionales de calidad medioambiental», elaborada por el gobierno sueco en 1998 y ratificada por el parlamento un año más tarde, y concebida como "sistema de gobierno por objetivos y resultados" como "medio más efectivo para dirigir una estrategia ampliamente concebida de DS», la integración sectorial figura como uno de los principios básicos para su implementación. Por integración sectorial se entiende la «incorporación (infusion) de responsabilidad para un DS ecológico en los cometidos (mandates) de agencias públicas y las actividades de empresas y otras organizaciones en distintos sectores sociales» (Lundqvist, 2002, p. 11 y 15). Tan interesante como la concreción del principio, que identifica tanto veinticuatro agencias públicas (cerca del 10\% del total) como la obligación para las mismas de realizar auditorías de gestión medioambiental, resulta el hecho de añadir el término ecológico a la estrategia de DS. Esta cualificación apunta a la ya mencionada necesidad de dar prioridad al componente medioambiental, al menos en los países desarrollados, en las estrategias de sostenibilidad. 
- La organización de un proceso de diálogo y consulta con los principales actores y con la ciudadanía en general (fórum).

- La formulación de una estrategia sectorial de cambio que incorpore principios básicos, objetivos y calendarios (estrategia).

- La formulación de un plan de acción sectorial en el que coincidan los objetivos señalados como prioritarios con los objetivos de las distintas políticas, y en el que se elijan los actores responsables para llevarlos a cabo (plan de acción).

- La integración del plan de acción en el presupuesto, así como en todos los otros fondos asignados al ministerio (contabilidad verde).

- El desarrollo de un sistema de control de los impactos, de los procesos de implementación y de los resultados en cuanto a objetivos que incorpore períodos de elaboración de informes de evaluación, revisión de la estrategia sectorial y plan de acción (programa de supervisión).

A pesar de la utilidad analítica que entraña la distinción entre integración horizontal y vertical, en la práctica ambos conceptos se presentan frecuentemente entremezclados. El primer Plan Nacional de Política Medioambiental, aprobado por el parlamento holandés en 1989, por ejemplo, define la integración sectorial (o vertical) como el «reforzamiento de la coherencia en el proceso de elaboración política» de forma que se "promueva la incorporación de consideraciones medioambientales en los ministerios sectoriales a través de extensos procesos de consulta y negociación, tanto interdepartamental como transsectorial» (Lundqvist, 2002, p. 21), lo cual implica una relativa yuxtaposición de las dos dimensiones de la integración.

En una investigación comparada acerca de la aplicación del DS y en la que se analizaba, entre otros, el estado en el que se encontraba el principio de integración, se concluyó que, en relación con la integración intraministerial (o dimensión vertical), el avance ha sido mas formal (y retórico) que sustantivo, ya que el elemento medioambiental es continuamente relegado a un segundo término en favor de los intereses desarrollistas (Lafferty, 2002). Con respecto a la integración horizontal, se señalaba que el estudio de las estrategias nacionales de DS es crucial, porque proporciona información sobre el tipo de compromiso político en torno a la sostenibilidad. Entre otras cosas, estas estrategias deberían reconocer abiertamente el fuerte potencial de conflicto que existe a la hora de intentar equilibrar los tres principios que componen el DS, algo que, por ejemplo, la Estrategia Española de Desarrollo Sostenible (EEDS), debido a lo que se ha denominado "vicio de la autocomplacencia", no hace, ya que recalca que la estrategia deberá acomodarse a las políticas económicas y sociales vigentes (Aguilar, 2002) ${ }^{8}$. Otro asunto de gran relevancia en estas estrate-

8. Por ello, únicamente desde una perspectiva retórica es posible entender las declaraciones del anterior ministro de Medio Ambiente español, Jaume Matas, tras la Cumbre de Johanesburgo, en las que abogaba por profundizar en temas como la integración del medio ambiente en las políticas de desarrollo económico. 
gias es la existencia o no de una autoridad central investida con un mandato de promoción de la sostenibilidad, la cual debería estar por encima de los ministerios o departamentos sectoriales, o bien porque pertenece al core executive (tal y como se intentó en el caso noruego), o porque se ha creado, ex novo, como una agencia de planificación (como ocurrió en las primeras fases de la estrategia holandesa), o porque se sitúa en el poder legislativo (caso de Canadá) ${ }^{9}$. $Y$ es que, aunque es difícil determinar a priori qué ministerios tienen más peso en las negociaciones internas sobre DS, es muy improbable que los ministerios de medio ambiente puedan, con relativa independencia del contexto geográfico, imponer sus tesis favorables al principio de integración sobre otros departamentos más poderosos y de mayor tradición.

En última instancia, es muy difícil empíricamente encontrar las dos dimensiones del principio de integración al mismo tiempo. En líneas generales, la dimensión vertical es la que más ha avanzado, mientras que la horizontal se encuentra en un estado embrionario, lo cual no es extraño si se considera que la primera implica un menor grado de conflicto interdepartamental y menos cambios drásticos con respecto a la asignación de beneficios y costes, y a la consiguiente aparición de ganadores y perdedores.

\section{El principio de integración en el entramado europeo}

Además de la multiplicidad de actores, individuales y colectivos, públicos y privados, que tienen que interiorizar el principio de integración, éste tiene que ser percibido como parte integrante de la estrategia que conduce a la mejora de la política medioambiental actualmente existente, o a la consolidación del DS, a través, en primer lugar, de su reconocimiento explícito en informes, comunicados y declaraciones (tanto de la Comisión, el Parlamento, el Consejo Europeo, o incluso la Agencia Europea de Medio Ambiente - AEM-, a nivel comunitario, como de la ONU y las cumbres mundiales que auspicia, por ejemplo, la recientemente organizada en Johanesburgo, o las reuniones de la Organización Mundial de Comercio - OMC_- el G-7 y otros organismos a nivel internacional). Este reconocimiento, que corresponde al nivel retórico-simbólico de la política, debe venir acompañado necesariamente, y en segundo lugar, de la institucionalización del principio, algo que sucede cuando el mismo pasa a formar parte del comportamiento cotidiano de individuos y grupos y del funcionamiento habitual de las instituciones. Sólo cuando el principio de integración se ha incorporado a la práctica diaria de los agentes públicos y privados puede pasarse a una tercera fase de operacionalización que persigue medir el éxito en su implantación a través de su control (monitoring) y evaluación.

9. Con respecto a tres estudios sobre governance para el DS encargados por la OCDE, se puede afirmar que el principio de integración ha sido incorporado en mayor medida por Canadá, en menor medida por el Reino Unido, situándose Alemania en una posición intermedia (Lafferty, 2002). 
A primera vista, la naturaleza del principio de integración no resulta ser la más indicada para que el mismo "triunfe» dentro de la UE. Precisamente porque la UE ha sido definida como gobierno, o polity, multinivel, con competencias políticas superpuestas entre múltiples niveles, y la posibilidad de coordinar muy distintas instancias de forma que todas interioricen en sus propuestas el componente medioambiental parece difícil de conseguir. Esta dificultad, en términos generales, debe ser matizada cuando se desciende en el nivel de concreción de análisis, ya que el alcance en que el principio de integración sirva como elemento operativo en el funcionamiento cotidiano de la UE variará dependiendo de la institución en cuestión.

El Consejo Europeo, por ejemplo, ha prestado gran atención al principio, tal y como lo demuestra la Iniciativa de Cardiff, pero esta atención se centra sobre todo en el aspecto retórico y de compromiso genérico hacia la integración. El problema de esta institución es que dedica poco tiempo a los detalles concretos de sus compromisos o estrategias y no se implica en la aplicación de los objetivos que se recogen en las declaraciones de las cumbres. Por ejemplo, los jefes de estado y gobierno reunidos en la Cumbre de Gotenburgo acordaron que todas las dimensiones del DS deberían ser revisadas en el contexto de la reunión anual de primavera que celebra el Consejo, pero delegaron en la Comisión la tarea de presentar un informe de síntesis que evaluara el progreso hacia la sostenibilidad en cada una de estas reuniones ${ }^{10}$. Además, dentro del Consejo, los diferentes grados de institucionalización del principio de integración tienen más que ver con factores sectoriales (qué ministros se reúnen en el Consejo) que con razones de nacionalidad. Es decir, los ministros de Medio Ambiente tienen, lógicamente, un mayor interés que cualquier otra figura política en promover principios que, como el de integración, ayudan a interiorizar la perspectiva conservacionista en el funcionamiento de la administración comunitaria y nacional. Es más, aquellos ministros más comprometidos con el tema (por la mayor sensibilidad ecológica de sus electorados o por su pertenencia a formaciones políticas verdes) han conseguido que, en el seno de las reuniones del Consejo, se aprobaran ambiciosas medidas que habrían encontrado serias resistencias en sus propios gobiernos, dentro de los cuales habrían tenido que enfrentarse, con escasas probabilidades de éxito, a ministerios «antiecológicos» de gran peso, como, por ejemplo, el de Industria y Energía. Además, la estructura sectorial de esta institución ha resultado, entre otras cosas, en que las recomendaciones para la mejor aplicación de las estrategias de DS y, consiguientemente, puesta en práctica del principio de integración, varíen según cuáles sean los ministros afectados. Así, el informe de marzo del año 2002 del Ecofin (Consejo de Economía y Finanzas) puso el énfasis en la necesidad de asegurar un mayor grado de flexibilidad a los estados miembros a la hora

10. Este cometido no parece haber sido resuelto por la Comision de forma satisfactoria, ya que el documento que preparó para la Cumbre de Barcelona, en el que no aparecían indicadores medioambientales, mereció la crítica unánime de la AEM, el Bureau Europeo de Medio Ambiente y de varias $\mathrm{ONG}$, como Amigos de la Tierra. 
de elegir y diseñar distintos tipos de instrumentos económicos para la protección del medio ambiente (en contraposición a la inflexibilidad que caracteriza a los instrumentos regulativos) (Lundqvist, 2002) ${ }^{11}$.

Ahora bien, aunque las razones sectoriales parecen tener un mayor peso que las identidades nacionales a la hora de promover tanto el principio de integración como otros vinculados al DS, la ausencia de medios que caracteriza al Consejo a la hora de hacer el seguimiento de sus propuestas implica que el compromiso hacia las mismas dependa, en gran medida, de las prioridades que marque el país que asume la presidencia semianual de esta institución. Ello se ve claramente en el caso de la Cumbre de Barcelona, que, anunciada por el director de la AEM como una ocasión histórica para que las exigencias medioambientales fueran finalmente integradas en condiciones de igualdad en la ambiciosa agenda socioeconómica que el Consejo aprobó en Lisboa en el año 2000, terminó con un resultado decepcionante, ya que los temas de DS, que inicialmente aparecieron como objetivos de la Presidencia española, terminaron siendo relegados a un segundo plano a favor de un enfoque centrado en la competitividad económica ${ }^{12}$. Debido a ello, y aunque se ha defendido recientemente que la tradicional distinción entre promotores (forerunners) y obstaculizadores (laggards) de la "causa» medioambiental es más que cuestionable (Kousis y Eder, 2001), porque los países tienen distintas prioridades medioambientales y no son consistentemente «verdes» en todos los ámbitos de esta política y porque, además, los estados miembros que generalmente han merecido el segundo rótulo han comenzado a defender sus propias agendas medioambientales con mayor vehemencia en la UE (Aguilar, 1997b), es indudable que la atención que se concede a los temas de medio ambiente varía significativamente según qué país ocupe la presidencia del Consejo. Lógicamente, por lo tanto, los avances que a nivel comunitario se produzcan en el principio de integración, o, de forma más global, en el programa de DS, dependerán en gran medida de la "sensibilidad ecológica» de los distintos países, lo cual da lugar a un movimiento pendular que oscila entre el optimismo (en cumbres como las de Cardiff de 1998, o Gotenburgo de 2001, en las que la sostenibilidad ocupó una posición clave) y el pesimismo (caso de la cumbre de Barcelona del 2002, en la que el tema pasó desapercibido).

La Comisión es probablemente la institución europea que más ha progresado en cuanto a la internacionalización del principio de integración en su funcionamiento cotidiano, aunque aquí hay que distinguir entre las diferentes

11. Se ha recomendado al Consejo Europeo, precisamente por su carácter sectorial, que cambie sus estructuras como manera de mejorar la coordinación y consistencia de su trabajo en las reuniones sectoriales.

12. El DS fue contemplado como uno de los objetivos del gobierno del PP cuando asumió la Presidencia del Consejo Europeo a comienzos del año 2002, pero las referencias al mismo fueron eliminadas en posteriores documentos a favor de temas como la lucha contra el terrorismo internacional, el desarrollo de la política exterior europea o la liberalización del mercado eléctrico (Aguilar, 2002). 
direcciones generales que la componen. Aunque, como es de sobra conocido, la Comisión trabaja en numerosas ocasiones siguiendo las peticiones o recomendaciones del Consejo, su margen de iniciativa legislativa es muy grande. Esta iniciativa se demostró, por ejemplo, en la elaboración de los diferentes informes sectoriales que dimanan del Proceso de Cardiff, los cuales delimitan la estrategia del Consejo a la hora de incorporar las preocupaciones medioambientales en otras actividades sectoriales. Otro caso reciente en el que la Comisión ha adoptado un enfoque activo en la promoción del DS ha sido su compromiso relativo a asegurar que todas sus propuestas incluyan una evaluación de impacto de sostenibilidad (sustainability impact assessment) que cubra las potenciales consecuencias económicas, sociales y medioambientales de las mismas - estas propuestas serán, además, redactadas por expertos externos, hechas públicas y contendrán una estimación presupuestaria-. A pesar de este progreso, la fragmentación horizontal que caracteriza a la Comisión, dividida tal y como está en distintas direcciones generales con diferentes objetivos y «clientelas», provoca indudables problemas a la hora de aplicar el principio de integración. La DG XI ha visto así frustrados sus intentos de consolidar el principio, ya que las otras direcciones generales no manejan, o lo hacen de forma insuficiente, la noción de integración. Por ejemplo, a finales del año 2001, la DG de Mercado Interno propuso reglas de contratación pública (public procurement) que dejaban poco espacio a la consideración de aspectos medioambientales, desoyendo la protesta de la DG XI, la cual recalcó que este tipo de integración era precisamente el que promovían el Proceso de Cardiff y el artículo $6^{\circ}$ del Tratado de Amsterdam. Dentro de esta línea crítica de actuación, los euroburócratas de la DG XI han aireado el hecho de que nadie dentro de la dirección general encargada de temas agrícolas esté dispuesto a hablar acerca de los problemas medioambientales que la Política Agrícola Común (PAC) provoca (Bomberg, 2002). Hay muchos otros ejemplos de no aplicación del principio de integración dentro de la Comisión, tanto en política pesquera, como de turismo o energía, aunque recientemente se han detectado algunas mejoras en cuanto a los mecanismos de asignación de fondos estructurales.

Por otro lado, un problema a añadir a la naturaleza fragmentada de la Comisión en cuanto a la promoción del principio de integración estriba en que su «activismo político y legislativo» se ha visto últimamente atemperado por el recelo de determinados países que, como es el caso del Reino Unido y Dinamarca, han protestado, aunque desde concepciones distintas acerca del significado del principio de subsidiariedad, por la supuesta injerencia de esta institución en asuntos que escaparían a su competencia ${ }^{13}$. El período posterior a Maastricht

13. Como la subsidiariedad está todavía lejos de haber alcanzado una definición unánime, ha sido defendida por países que tienen diferentes (si no divergentes) objetivos en protección medioambiental. El Reino Unido, por ejemplo, ha vinculado el principio a la idea de que esta política debe volver a ser tarea de los estados miembros. Esta postura de «renacionalización» se explica, entre otras razones, por la oposición británica al enfoque comunitario de (homogéneos y costosos) estándares de emisión. Al mismo tiempo, la subsidiariedad ha 
está vinculado estrechamente al principio de subsidiariedad, aunque el hecho de que el Tratado de Amsterdam establezca las reglas para su aplicación en un protocolo ha sido interpretado como una forma de limitarlo. A pesar de ello, la Comisión está intentando disipar las dudas que rodean la legitimidad de su actuación en la política medioambiental mediante un mayor énfasis en el diálogo y la cooperación entre las instituciones comunitarias, los estados miembros, las industrias y los ciudadanos (siguiendo el concepto de responsabilidad compartida). Esta institución sopesa ahora más detenidamente la conveniencia o no de proponer nueva legislación medioambiental y se ha propuesto reducir su actividad regulativa. En la actualidad, por lo tanto, la mayoría de esta actividad se dirige a la actualización de la legislación existente o al desarrollo de directivas marco, al tiempo que, en el proceso de preparación de directivas, se han aumentado las consultas con distintos grupos y se ha intensificado el papel del Parlamento Europeo. No sólo la subsidiariedad sino también sonados fracasos políticos, como el intento fallido por parte de la Comisión de adoptar un impuesto energético, están detrás de esta mayor cautela (Grant y otros, 2000). Este menor activismo legislativo, que encaja perfectamente en el escenario político de la desregulación y de la retirada del estado de determinados ámbitos, fue ya promovido por Delors desde finales de los años ochenta y asumido por los sucesivos presidentes de la Comisión Europea. A pesar de que este escenario es desfavorable para la activa maquinaria administrativa de Bruselas, la Comisión percibe el DS como una tarea en expansión que puede y debe ser explotada. Su compromiso con este tema tiene mucho que ver con la existencia dentro de esta institución de figuras similares a lo que la literatura sobre movimientos sociales denomina «empresarios políticos»: personas que dentro de la sociedad civil son capaces de generar recursos (tanto políticos como económicos) que después utilizan para hacer avanzar sus propuestas o tesis. En el caso del DS, tales personas trabajarían dentro de la administración, principalmente en la DG XI, pero también en los círculos próximos al presidente de la Comisión.

El Parlamento Europeo (PE) es la institución comunitaria que más ha hecho por promover el DS y el principio de integración. Como esta instancia no tiene

sido promovida por países, como Dinamarca, que no se resisten a esa estandarización sino que temen, por el contrario, que la política medioambiental pueda derivar hacia prácticas de «mínimo común denominador» que satisfagan las exigencias de los estados miembros menos comprometidos con el tema. Curiosamente, por lo tanto, el mismo principio ha sido apoyado por países sin un claro perfil ecologista (Reino Unido), y por otros que, implicados en una rigurosa protección del medio ambiente, temen que la política comunitaria pueda suponer un retroceso con respecto a los logros alcanzados a nivel nacional (Dinamarca). A pesar de estas diferencias, las exigencias de ambos grupos de países han terminado convergiendo: se pide así que la política medioambiental sea «devuelta» (en el sentido del término devolution) a los estados, y que la Comisión atempere su activismo legislativo (Aguilar, 1997b). Esta convergencia no es óbice para que la probabilidad de éxito de hipotéticas denuncias de la Comisión ante el Tribunal de Justicia Europeo por parte de países que argumentan el quebranto de este principio en el tema medioambiental sea por ahora pequeña (Lodge, 1994). 
capacidad de iniciativa legal, su influencia se limita a sus comentarios, recomendaciones, críticas y aprobación o rechazo a informes, programas y propuestas de directivas, principalmente. El PE criticó, por ejemplo, la Estrategia de DS de la Comisión por no incorporar suficientemente el principio de integración en sus procedimientos, e impulsó diversas enmiendas encaminadas a la promoción del mismo, entre ellas la que sugería reorganizar la Comisión de forma que los requisitos medioambientales fueran integrados en todos sus niveles. En la etapa previa a la celebración de la Cumbre de Barcelona, la cámara aprobó una resolución, por una impresionante mayoría de 490 votos, en la que exigía que la Comisión y el Consejo se atuvieran a su compromiso a favor de la integración como medio de avanzar hacia el DS. A la hora de entender el porqué de este empeño, es importante tener en cuenta la dinámica de competencia institucional que caracteriza a la UE y que hace del PE la única instancia comunitaria que cuenta con lo que podría denominarse «legitimidad democrática supranacional», en el sentido de estar compuesta por personas elegidas en procesos electorales de ámbito nacional que se integran en formaciones políticas, sin embargo, de ámbito europeo. Esta legitimidad, y la hasta ahora laxa ligazón de la cámara a intereses domésticos, la convierten en una institución consagrada al avance del proyecto europeo, parte fundamental del cual es el progreso en la protección del medio ambiente a nivel comunitario, tarea a la cual los ciudadanos europeos, tal y como lo reflejan los distintos eurobarómetros, conceden un gran apoyo. A pesar de todo ello, también se han hecho recomendaciones al PE para que optimice su funcionamiento con vistas a la promoción de la sostenibilidad, señalándose que debería establecer un comité de DS, con representantes de otros comités, para mejorar la integración de los aspectos medioambientales en el proceso decisorio (Lundqvist, 2002).

El Tribunal de Justicia Europeo (TJE) ha demostrado, a través de determinadas sentencias, tal vez la más conocida sea la de las «botellas danesas», que el componente medioambiental puede estar por encima de los principios básicos del mercado común. En el caso de las «botellas danesas» (caso 302/86), en el que un sistema de depósito y retorno de envases establecía determinados requisitos a cumplir por los productores y distribuidores de cervezas y refrescos, se aceptó el sistema introducido por Dinamarca (dentro de la doctrina de la «integración diversificada») y se desestimó la denuncia de la Comisión, porque la protección del medio ambiente constituía un objetivo obligatorio que podía limitar la aplicación del artículo 30 del Tratado de Roma sobre la libre circulación de mercancías ${ }^{14}$. Además, se argumentó que las restricciones que el

14. Un año antes, en 1985, el TJE sentenció en la misma línea que Francia estaba legitimada para crear sistemas restrictivos de recogida y tratamiento de aceites usados (siguiendo la DIR 75/439), siempre y cuando éstos aseguraran que la eliminación de tal desecho se realizaba de forma que evitara perjuicios al medio ambiente. Por ello, el principio de libertad de comercio no sería absoluto, sino que estaría sujeto a ciertos límites que, a pesar de justificarse por razones de interés general, no debían ser ni discriminatorios ni desproporcionados (Krämer, 1988). 
sistema imponía al movimiento de bienes no eran desproporcionadas con respecto al fin medioambiental. Este caso marcó un hito, ya que la Comisión había señalado, antes de que se conociera la sentencia, la necesidad de determinar «hasta qué punto la preocupación por la protección del medio ambiente [tenía] prioridad sobre el principio del mercado común sin fronteras ya que [existía] el riesgo de que (determinados) estados miembros pudieran buscar refugio detrás de argumentos ecológicos para evitar abrir sus mercados» (Koppen, 1992 , p. 20). Frente a estas sentencias favorables al medio ambiente, el principio de reconocimiento mutuo (elaborado a raíz del caso Cassis de Dijon) podría tener repercusiones adversas para el mismo. La liberalización, o des-regulación, que acompaña a este principio tiene dos consecuencias diferentes: por un lado, simplifica el largo y costoso proceso de armonización que intenta establecer estándares europeos uniformes y detallados, mientras que, por el otro, puede facilitar la entrada de bienes de "baja calidad ecológica» en países con niveles estrictos de protección del medio ambiente (Aguilar, 1997a). Junto a las sentencias comentadas, que claramente favorecen la «causa» medioambiental, el TJE puede alentar el DS al promover el principio de integración, y dar prioridad, como ya ha hecho, a las exigencias conservacionistas siempre y cuando sean percibidas como legítimas y no como intentos encubiertos de proteccionismo comercial.

Aunque la Agencia Europea de Medio Ambiente (AEM) no forma parte strictu sensu del entramado comunitario ${ }^{15}$, es una institución importante encargada de evaluar la política medioambiental y de recordar incesantemente, tanto a las distintas instancias que componen la UE como a los países miembros, sus compromisos en DS. Con el objetivo de progresar en el desarrollo de esos compromisos, la AEM ha defendido incesantemente el principio de integración y ha elaborado numerosos trabajos que informan sobre la existencia o no de avances en torno al mismo. Como ya se vio anteriormente, el documento que la Comisión Europea preparó para la Cumbre de Barcelona, en respuesta al mandato recibido por el Consejo en cuanto a la necesidad de hacer un seguimiento de los avances y retrocesos en torno a la estrategia de sostenibilidad, fue enérgicamente criticado por la AEM, entre otros actores, por la premura con que se había preparado y por su deficiente contenido, en el que no aparecían, por ejemplo, indicadores medioambientales.

\section{Conclusiones}

Dentro de la UE, el principio de integración fue concebido inicialmente como la incorporación del componente medioambiental a todas aquellas actividades sectoriales que tenían efectos negativos sobre el entorno, con el objetivo

15. Junto al entramado institucional de la UE existen tambien actores de otro tipo, principalmente varias $\mathrm{ONG}$, que se encargan de propagar las virtudes del principio de integración, como el Bureau Medioambiental Europeo, una federación de 133 organizaciones ecologistas de países de dentro y fuera de la Comunidad. 
último de mejorar los rendimientos de la política de protección del medio ambiente a nivel comunitario. Posteriormente, el principio se ha vinculado a la estrategia europea de sostenibilidad, de modo que, actualmente, se defiende la compatibilidad entre crecimiento económico, conservación del medio físico y bienestar social. Esta "versión ampliada» de la integración disimula los conflictos de interés que se desatan cuando se intenta aplicar el DS, ya que la teórica armonía entre estos tres elementos es falsa y, por ello, es siempre necesario dar prioridad (en el tiempo, al menos) a alguno de los mismos. No hay nada que objetar, pues, a tal necesidad de priorización, siempre y cuando se haga explícita y se expliquen las razones que han conducido a la misma, así como las consecuencias de tal elección. Para algunos autores, el componente medioambiental debe, sin embargo, ser el elegido en las estrategias de sostenibilidad que todavía están elaborando la mayoría de los países desarrollados, porque es en la conservación del entorno donde menos progresos se han constatado y donde mayores pérdidas irreversibles pueden producirse. La dimensión horizontal del principio de integración que se refleja en estas estrategias está todavía, en líneas generales, poco desarrollada, mientras que la dimensión vertical (o intradepartamental) ha experimentado distintos avances según el país en cuestión, aunque, de forma genérica, se ha asentado principalmente en el nivel retórico-formal de la política y no en el sustantivo.

A nivel comunitario, el principio de integración parece haber progresado principalmente en el seno de la Comisión, aunque su naturaleza, como organismo caracterizado por su fragmentación horizontal, supone un freno importante a la hora de conseguir su institucionalización. El Consejo, por su parte, ha asumido el principio en sus declaraciones, pero la atención que le concede depende, en gran medida, del país que asume la presidencia semianual. Con respecto al Tribunal de Justicia, todavía habrá que esperar a futuras sentencias para determinar cuál es la relevancia que esta institución otorga a la integración. Es pues el Parlamento la instancia comunitaria que, de manera más sistemática, ha empujado a favor de la incorporación del principio de integración, tanto a nivel europeo como de los países miembros. Y es que este principio, tanto si se entiende (como se hacía en un primer momento) como mecanismo que contribuye a mejorar los resultados de la política medioambiental, o a reducir su déficit de implementación, como si se concibe (en el momento actual) como mecanismo que obliga a considerar en situación de igualdad (cuando no, dándole prioridad) al componente del medio ambiente frente a los otros dos que configuran la sostenibilidad dentro de las estrategias de DS, es imprescindible a la hora de progresar en la lucha contra el deterioro ecológico, tarea que, como bien saben los eurodiputados, goza de gran popularidad entre los ciudadanos de la UE. 


\section{Bibliografía}

Aguilar, S. (1997a). El reto del medio ambiente. Conflictos e intereses en la política medioambiental europea. Madrid: Alianza Universidad.

- (1997b). "Subsidiarity, Shared Responsibility, and Environmental Policy in Spain». En Collier, U.; Golub, J.; Kreher, A. (eds.). Subsidiarity and Shared Resposibility: New Challenges for EU Environmental Policy. Baden-Baden: Nomos Verlagsgesellschaft.

- (2002). «Sustainability is Cool: Rhetorical Participatory Discourse in the Spanish Strategy for Sustainable Development». Comunicación presentada en el seminario organizado por ProSus sobre Governance of Sustainable Development (Roma, noviembre de 2002).

BOMBERG, E. (2002). «Sustainable Development and Regional Governance: Multilevel Mechanisms and Coordination in the European Union». Comunicación presentada en el seminario organizado por ProSus sobre Governance of Sustainable Development (Lillehammer).

Grant, W.; MatTHews, D.; Newell, P. (2000). The Effectiveness of European Union Environmental Policy. Londres: MacMillan.

Koppen, I.J. (1992). "The Role of the European Court of Justice in the Development of the European Community Environmental Policy». EUI WP n. 92/18. Florencia.

KRÄMER, L. (1988). «Einheitliche Europäische Akte und Umweltschutz: Überlegungen zu einigen neuen Bestimmungen im Gemeinschaftsrecht». En RENGELING, H.W. Europäisches Umweltrecht und europäische Umweltpolitik. Berlín: Carl Heymanns.

LAFFERTY, W. (2002). «From Environmental Protection to Sustainable Development. The Challenge of De-coupling through Sectoral Integration». Comunicación presentada en el seminario organizado por ProSus sobre Governance of Sustainable Development (Lillehammer).

Lodge, J. (1994). The European Community and the Challenge of the Future. Londres: Frances Pinter.

LUNDQVIST, L. (2002). «Management by Objectives. A Comparison of Dutch, Swedish and EU Strategies for SD». Comunicación presentada en el seminario organizado por ProSus sobre Governance of Sustainable Development (Rome).

RaYner, S. (1994). "Governance and the Global Commons». The Centre for the Study of Global Governance. London School of Economics. Discussion Paper 8. 